\title{
Liver transplantation and primary biliary cirrhosis
}

\author{
A.K. Burroughs, M. Biagini' ${ }^{1}$ P.A. McCormick and K. Rolles \\ Hepato-biliary and Liver Transplantation Unit, Royal Free Hospital and School of Medicine, London NW3 2QG, \\ UK, and ${ }^{1}$ Policlinico di Careggi, Servizio Autonomo di Gastroenterologia, Firenze, Italy.
}

\section{Introduction}

Primary biliary cirrhosis (PBC) is a chronic cholestatic liver disease, autoimmune in 'nature', but of unknown aetiology. ${ }^{1,2}$ It often presents in middle-aged women with itching, jaundice, hepatomegaly or variceal bleeding. ${ }^{3}$ Increasingly the diagnosis is made in asymptomatic patients or those without symptoms referrable to the liver, ${ }^{4}$ often in association with other autoimmune diseases, on the basis of abnormalities in liver function tests, particularly alkaline phosphatase and the presence of mitochondrial antibody which is found in $95 \%$ of patients. ${ }^{5}$ The disease is usually progressive especially when pruritus and other liver based symptoms are present. Chronic cholestasis, fibrosis, cirrhosis and portal hypertension supervene and the cause of death is due to hepatic failure, bleeding varices, or sepsis in $85 \%$ of cases.

Long standing jaundice leads to poor absorption of fat soluble vitamins $A, D, K$ and $E$, as well as calcium and phosphate. An important complication of this is progressive cortical bone thinning leading to osteoporosis. This is made worse by the perimenopausal clinical setting. ${ }^{6}$ Prophylactic vitamin $D$ and calcium supplementation whilst preventing osteomalacia does not necessarily retard the onset of osteoporosis and its complications. ${ }^{6}$ Significant malnutrition can occur in the later stages of the disease. Pruritus usually responds to cholestyramine, but in some cases intractable itching produces an unacceptable quality of life and is very difficult to treat. Variceal bleeding is usually treated by sclerotherapy but the risk of rebleeding is still approximately $50 \%$ despite repeated injections as for other types of cirrhosis. Ascites develops late and, in the presence of severe cholestasis, is often difficult to treat and becomes resistant to diuretics.

Numerous drugs for the specific treatment of PBC have been evaluated in placebo controlled studies: azathioprine ${ }^{7,8}$ chlorambucil, ${ }^{9}$ colchicine, ${ }^{10-12}$ cyclosporin A, ${ }^{13,14}$ D-penicillamine, ${ }^{15-22}$ prednisolone ${ }^{23}$ and

Received:15 February 1989

Correspondence: A.K. Burroughs, M.B., Ch.B., M.R.C.P. ursodeoxycholic acid, ${ }^{24}$ to establish if the progression of the disease can be stopped or slowed down.

When considering liver transplantation for PBC against this background, and in particular for any one patient, there are 5 questions to be asked: (1) what is the natural history of PBC?; (2) is there effective specific medical treatment?; (3) what does liver transplantation achieve?; (4) what is the patient's attitude towards the consequences of the disease and to liver transplantation?; (5) what is the optimal timing for a liver transplant?

\section{What is the natural history of PBC?}

Some authors have made a distinction between asymptomatic and symptomatic $\mathrm{PBC}^{25}$ suggesting that asymptomatic disease does not result in an increased mortality compared to age-matched women in the general population. However, it is likely that women with $\mathrm{PBC}$ are being diagnosed earlier on in the course of their disease, and this selection bias only leads to an apparent improvement in survival. Use of screening leads to more patients with a very slow rate of progression being recognized, and may lead to selection which is not representative of the total population of PBC ${ }^{26}$ Asymptomatic patients may die from liver disease $^{26}$ and their course follows that of symptomatic patients once they develop symptoms, ${ }^{26-28}$ suggesting overall prognosis is not good. ${ }^{26-28}$ It follows that only identification of asymptomatic patients who will progress, will allow separation of a sub-group of PBC patients with a good long-term prognosis.

There is far less disagreement about the course of PBC patients with symptoms, and their markers of disease progression. Serum bilirubin had been identified by a number of authors, ${ }^{8,29-30}$ and serial studies have shown that once serum bilirubin values rise to above $34 \mu \mathrm{mol} / 1$ only half the patients will live to 49 months (95\% confidence intervals $32-74$ months), ${ }^{29}$ once values reach $100 \mu \mathrm{mol}$, half the patients are dead within 2-3 years. However, these 
estimates are too imprecise: $\mathrm{PBC}$ patients may die from liver disease with low bilirubin values. Histological stage is important, cirrhosis and central cholestasis being associated with a worse prognosis. ${ }^{8}$ In a serial histological study $88 \%$ of patients had cirrhosis 1 year before death. ${ }^{31}$ Again this single variable is too imprecise as a prognostic marker, as ascites and variceal bleeding do not correlate well. ${ }^{8}$ Increasing age is clearly an important risk factor ${ }^{8,30}$ but sex is not ${ }^{8,30,32,33}$ although male patients have an increased risk of developing hepatocellular carcinoma $(33 \% v s 5 \%)^{34}$ as in all other forms of cirrhosis.

Screening programmes with serial ultrasonography and serum alpha-fetoprotein measurements may detect asymptomatic hepatocellular carcinomas, but these have yet to be shown to alter the natural history in Western populations. It is intriguing that the Pittsburgh transplant group have found similar survival in those cirrhotics whose small hepatocellular carcinomata were found incidentally in the recipient liver compared to cirrhotics without such tumours. ${ }^{35}$ Larger, clinically manifest tumours recur following liver transplantation but some cases of a 5 year tumour-free interval are recorded ${ }^{36} \mathrm{PBC}$ patients also have a higher incidence of other malignant tumours particularly breast cancer ${ }^{37,38}$ and cancer accounts for $8 \%$ of PBC deaths in one series. ${ }^{8}$ Extrahepatic metastases of hepatocellular carcinoma and extra-hepatic malignancy are a contraindication to liver transplantation. In addition patients with large symptomatic tumours do not usually do well following a liver transplant. ${ }^{36}$ Thus the development of malignancy in PBC which can occur before the onset of liver failure precludes transplantation in some patients. At present the development of malignancy cannot be predicted.

\section{Is there an effective specific medical treatment for PBC?}

Since 1985, twelve randomized controlled trials of specific therapy have been published. Recently these have been reviewed in detail ${ }^{39}$ with a conclusion that no single drug is wholly effective. No drug has been demonstrated to halt histological progression. No drug except cyclosporin A in non-cirrhotic $\mathrm{PBC}^{13}$ has improved both symptoms and biochemistry and only azathioprine appears to increase survival. ${ }^{8}$ However, this is only by an average of 20 months, and is estimated (using a Cox model) with $25 \%$ of the trial population being lost to follow-up. Furthermore, all of the randomized studies have some serious methodological flaws, ${ }^{39}$ the most important of which is insufficient sample size to assess a modest therapeutic advantage. This was appreciated by Epstein et al ${ }^{40}$ when evaluating D-penicillamine. They calculated that in order to detect an improvement from $60 \%$ to $70 \%$ in 5 year survival, approximately 750 patients would be required - more than the total number in the 8 randomized studies published to date! Colchicine ${ }^{10-12}$ and ursodeoxycholic acid ${ }^{24}$ improve biochemistry but when the latter is stopped biochemical values return to those seen in the placebo group. Whether these 'choleretic' properties can modify progression of the disease remains to be seen.

In the future, effective drug therapy for PBC may result from a better understanding of the immunological mechanisms which destroy bile ducts, but at present these are not well worked out. In our view and that of Wiesner et al..$^{39}$ it is important to continue to search for effective drugs but trials need to be well designed, executed and analysed, and will need to be multicentre to recruit sufficient patients. Wiesner et al. ${ }^{39}$ have suggested a 'reversible stage' before the onset of major destruction of bile ducts when immunosuppressive therapy may influence continuing bile duct necrosis, and an 'irreversible stage' when chronic cholestasis supervenes followed by fibrosis and cirrhosis. Possibly the anti-fibrotic drugs and bile acid therapy may be useful in halting or retarding fibrosis. When cirrhosis is present with portal hypertension or with major loss of bile ducts it is unlikely that any medical treatment can reverse this. Orthotopic liver transplantation is then the only therapeutic option.

\section{What does liver transplantation achieve?}

Primary biliary cirrhosis is the second most frequent indication for liver transplantation in adults. ${ }^{41}$ The most complete series of 76 patients has recently been reported from the Pittsburgh group, all of whom received cyclosporin $A$ as part of their immunosuppressive regimen.$^{42}$ Fifteen patients were over 50 years of age, and the mean bilirubin concentration was $342 \mu \mathrm{mol} / \mathrm{l}$ (range 25 to 867 ). Two thirds of patients had pruritus, two-fifths had fatigue, and nearly threequarters had ascites and a history of gastro-intestinal haemorrhage. The hepatectomies were easier than in other types of cirrhosis especially in the absence of previous abdominal surgery, although $20 \%$ of patients had an interposition graft using donor iliac artery because of intimal dissection of the recipient hepatic artery. Nineteen $(25 \%)$ required retransplantation (in 2 patients, 3 transplants were performed) 9 of whom survived making a total of 97 liver transplants in this series. Forty two other operations were needed for various complications. Despite this apparent 'heroic' surgery $52(68 \%)$ patients are alive between 1 and 6.5 years, with an actuarial 5 year survival rate of $66 \%$.

It is important to note that as with other indications for liver transplantation except malignancy, the survival curve is almost flat after the first year of survival. 
Most deaths occur in the first 3 months, and in this PBC series ${ }^{42}$ rejection accounted for half of the deaths. Rehabilitation is usually excellent. In this series, 17 are working full-time, 4 part-time and 28 at home able to care for themselves. Only two needed home care and one remained in hospital. None were jaundiced. This compared to only 1 working full-time, 2 part-time and 19 able to care for themselves at home before transplantation, when 54 were dependent on home or hospital care. However, some transplanted patients required 3 to 6 month stays in hospital because of previous bone disease before their functional status and their bone disease improved. ${ }^{43}$

Similar survival figures have been reported from European centres. ${ }^{44}$ Clearly the clinical reality is that transplantation improves survival and quality of life in PBC. As no randomized study without transplantation will be performed it is reassuring that the Mayo Clinic prognostic model (interestingly without histological stage) derived from 418 PBC patients ${ }^{45}$ when applied to 161 PBC transplants at Pittsburgh showed that at 1 year the estimated survival without transplantation was $45 \%$ whereas the actuarial survival rate with transplantation was $76 \%(P<0.001)$. In the long term the only potential problem is the possible recurrence of PBC, only reported by the Cambridge/King's College group. ${ }^{46,47}$ However, this is disputed ${ }^{48}$ as there are many histological similarities to rejection; no recurrence has been reported in Pittsburgh..$^{42}$ In any case it appears to be a very rare event and should not hinder a decision to transplant. The immunosuppression regimens post-transplant would probably modify any disease recurrence. However, it is clear that mitochondrial antibodies remain positive following transplantation. ${ }^{49}$

\section{What is the PBC patient's attitude towards the consequences of the disease and to liver transplantation?}

The patient's attitude towards the effects of the disease and the risks of transplantation are important in all areas of transplant medicine, and no less so for the patient with PBC. In some, the complications of the disease affect their quality of life so much that there is less concern with the risk of dying, in particular fatigue, pruritus, bone pain, recurrent bleeding, ascites and loss in weight or any combination thereof. Other patients may have psychological problems in realising that somebody has had to die in order to donate a liver, or may not be able to cope with a life-long dependence on immunosuppressive treatment. An assessment of the patients' attitudes must influence the clinician's decision and advice. In a similar setting where surgery for carcinoma of the lung entailed an immediate risk of death, or the possibility of long term survival, McNeil et al. ${ }^{50}$ have shown how patients' attitudes towards survival and risk-taking may be assessed. The patients were asked to choose amongst a hypothetical series of gambles between periods of certain survival and the chance of longer survival. From their choices it was possible to construct curves which quantified the value of outcome important to the patients themselves - the 'utility function' of clinical decision analysis. ${ }^{51}$ Some patients with PBC may be more risk seeking, if, for example, they have been made unemployable because of their disease, or have dependents, or if their particular life style has been altered substantially by the disease. Others who have not worked perhaps with no children at home may prefer to put up with the same symptoms as the 'risk-seekers' rather than take the risk of immediate death. Usually the patients' attitudes to the disease initially only reflect their symptoms. However, the prognostic information supplied by the clinician assumes an important role when speaking about the possibility of transplantation with the patient and her or his family. No detailed work about patients' attitudes to their chronic liver disease and to liver transplantation is presently published, although this is being evaluated at the Royal Free Hospital.

Clinicians involved in assessing a patient for liver transplantation should in effect gauge the patient's threshold for deciding to go ahead with a transplant (clearly after the risks have been explained) as well as their own threshold for advising the patient to have a transplant. This can be considered as the point of indifference, i.e. the outcome is the same either waiting or transplanting - similar in concept to the "threshold approach' in decision analysis. ${ }^{51}$

\section{What is the optimal timing for a liver transplant?}

Decision analysis, ${ }^{51}$ even with the recent advances ${ }^{52}$ is not particularly suitable in the context of liver transplantation as the diseases are inevitably fatal with the majority of the deaths being related to the disease,${ }^{8}$ in $\mathrm{PBC}$ at least $85 \%$. The question is not which treatment to choose, but when to use transplantation. There are now no specific contraindications to liver transplantation related solely to intrahepatic disease. Only severe cardio-pulmonary disease and chronic renal failure (in the absence of a dual or triple organ transplant), and extrahepatic sepsis or malignancy are considered absolute contraindications. ${ }^{41}$ Thus the following factors should be considered when advising liver transplantation: (1) the patient's threshold for going ahead with a transplant; (2) the patient's prognostic index; (3) the patient's risk factors for transplantation.

The patient's threshold for deciding to proceed with transplantation has been discussed in the previous section. 
The PBC patient's precise prognosis at any one time is difficult to determine, but progress has been made using Cox's multivariate regression models ${ }^{8,30,45}$ which result in a series of independently predictive variables for survival. An indication of their relative importance can also be assessed. From this, a prognostic index can be derived, and estimated survival times with confidence intervals can be graphically displayed. ${ }^{8}$ The best model published so $\mathrm{far}^{8}$ includes age, serum bilirubin, serum albumin, presence of cirrhosis, presence of central cholestasis and treatment with azathioprine. However, all these models are static (time independent) and do not take into account changes with time and more importantly the occurrence of potentially life threatening complications such as variceal bleeding, spontaneous bacterial peritonitis, nor the occurrence of malignancy which may preclude transplantation. These are competing risks over and above the decline in hepatocellular function. We believe that this concept should be extended to complications before transplant which may have a significant morbidity in the peri-operative period or post-transplant period, such as previous right upper quadrant or shunt surgery and severe bone disease.

Time dependent Cox modelling may solve some of these issues ${ }^{53}$ and the Mayo Clinic Group, the International Azathioprine Trial Group and ourselves at the Royal Free Hospital are developing these models for PBC. These should provide more precise estimates of survival to be established in any single patient. New markers of progression of disease may help in refining the prognostic index in the future, such as procollagen peptide III, ${ }^{54,55}$ hyaluronic acid, ${ }^{56}$ mitochondrial antibody subtypes ${ }^{57}$ and of, particular interest, quantitative liver function tests such as galactose elimination capacity. ${ }^{58}$ Serial measurements might allow a linear regression line to be drawn - in 5 patients when the line crossed the value of $4 \mathrm{mg} / \mathrm{min} / \mathrm{kg}$ the actual date of death was within 25 days. ${ }^{58}$ If this can be reproduced in much larger series, it will provide a valuable prognostic tool.

In practical terms patients with chronic liver disease without malignancy should be transplanted a year before the expected time of death, but other considerations related to the risk of the procedure come into the decision process. Thus a prognostic index for the risk of death following transplantation is not necessarily the same as that for the disease. Pre-operative risk factors in PBC patients have been assessed by Neuberger et al., ${ }^{59}$ univariate analysis showed that better prognosis following transplantation was associated with bilirubin concentration under $350 \mu \mathrm{mol} / 1$, prothrombin time under 5 seconds prolonged, serum sodium over $130 \mathrm{mmol} / \mathrm{l}$ and treatment with diuretics. In contrast age, blood group and previous abdominal surgery were not risk factors. A
Cox's model showed pre-operative serum bilirubin, serum urea, diuretic responsive ascites, and year of transplant (reflecting selection and a 'learning' curve) to be independently predictive. Although there was a correlation between the prognostic indices for the disease and for risk of death following transplantation, this was very weak $(r=0.55)$ which is not surprising as the major causes of death ${ }^{42}$ are rejection, acute graft non-function and hepatic artery thrombosis, which are difficult to relate to the severity of liver disease. Abnormal renal function prior to transplantation has also been found to be a significant adverse factor in other reports ${ }^{59-62}$ with a serum creatinine above $207 \mu \mathrm{mol} / 1$ predicting mortality in $79 \%$ of cases. ${ }^{60}$ Severe hyponatraemia may lead to central pontine myelinolysis, if rapidly corrected as may occur post transplant, so that gradual pre-operative correction should be attempted to achieve values about $125 \mathrm{mmol} / 1 .^{63,64}$ Interestingly, HLA matching and other immunological parameters prior to transplantation do not predict survival. ${ }^{65}$

Bismuth's group has set out selection criteria for transplantation in PBC, and reported their results, ${ }^{66} \mathrm{In}$ this group, out of $180 \mathrm{PBC}$ patients seen, the following criteria were taken as indications: serum bilirubin $>150 \mu \mathrm{mol} / \mathrm{l}$ and/or ascites and the presence of invalidating asthenia and/or pruritus. Contraindications were 'poor global condition' - including old age and severe extrahepatic diseases. In this group $50 \%$ did not meet the criteria. In the remainder, $38(22 \%$ of the total) had a contraindication and amongst the 52 $(28 \%)$ selected for transplant (48 had the decision based on objective criteria), 15 died waiting for a donor (all with worse liver function) and 37 received transplants with $85 \%$ survival. Clearly this group is different from the Pittsburgh series ${ }^{42}$ and demonstrates the difficulty in comparing results from different centres without detailed knowledge of the pretransplant condition of the patients.

\section{Conclusions}

At present orthotopic liver transplantation represents the only effective treatment for patients with advanced primary biliary cirrhosis. Presently prognostic models can predict the gradual hepatocellular failure (the predominant mode of death) far better than the risks of sudden death due to complications of the disease, such as first variceal bleeding, or the development of malignancy. In terms of transplantation these can be considered as competing risks versus death due to hepatocellular failure. More precise survival estimates to enable better timing of transplantation may be available with the more sophisticated time dependent Cox modelling, and improvements in assessing life expectancy using decision analysis. ${ }^{67}$ As risk factors 
related to early mortality (which accounts for $75 \%$ of all deaths) following liver transplantation become better understood and preventable, the risk of immediate death will be lessened. This will lower the patient's threshold to go ahead with a transplant. Once the patient with PBC has developed bilirubin

\section{References}

1. Sherlock, S. Primary biliary cirrhosis (chronic intrahepatic obstructive jaundice). Gastroenterology 1959 , 37: 574-596.

2. James, S.P., Hoofnagle, J.H., Strober, W \& Jones, E.A. Primary biliary cirrhosis: a model autoimmune disease. Ann Intern Med 1983, 99: 500-512.

3. Sherlock, S. \& Scheuer, P.J. The presentation and diagnosis of 100 patients with primary biliary cirrhosis. N Engl J Med 1973, 289: 674-678.

4. James, O., Macklon, A.F. \& Watson, A.J. Primary biliary cirrhosis - a revised clinical spectrum. Lancet 1981, i: $1278-1281$.

5. Kaplan, M.M. Primary biliary cirrhosis. N Engl J Med 1987, 316: 521-528.

6. Matloff, D.S., Kaplan, M.M., Neer, R.M., Goldberg, M.J., Butman, W. \& Wolfe, H.J. Osteoporosis in primary biliary cirrhosis: effect of 25 hydroxyvitamin D3 treatment. Gastroenterology 1982, 83: 97-102.

7. Heathcote, J., Ross, A. \& Sherlock, S. A prospective controlled tial of azathioprine in primary biliary cirrhosis. Gastroenterology 1976, 70: 656-660.

8. Christensen, E., Neuberger, J., Crowe, J. et al. Beneficial effect of azathioprine and prediction of prognosis in primary biliary cirrhosis. Final results of an international trial. Gastroenterologv 1985. 89: 1084-1091.

9. Hoofnagle, J.H., Davis, G.L., Schafer, D.F. et al. Randomized trial of chlorambucil for primary biliary cirrhosis. Gastroenterology 1986, 91: 1327-1334.

10. Kaplan, M.M., Alling, D.W., Zimmerman, H.J. et al. A prospective trial of colchicine for PBC. N Engl J Med 1986, 315: 1448-1454.

11. Warnes, T.W., Smith, A., Lee, F.I., Haboubi, N.Y., Johnson, P.J. \& Hunt, L. A controlled trial of colchicine in primary biliary cirrhosis. Trial design and preliminary report. J Hepatol 1987, 5: 1-7.

12. Bodenheimer, H., Schaffner, F. \& Pezzullo, J. Evaluation of colchicine therapy in PBC. Gastroenterology 1988, 95: 124-129.

13. Wiesner, R.H., Dickson, E.R., Lindor, K.D., Jorgenson, R., La Russo, N.F. \& Baldus, W. A controlled clinical trial evaluating $\mathrm{Cy}$ in the treatment of PBC: a preliminary report. Hepatology 1987, 7: 1025 (abstract).

14. Minuk, G.Y., Bohme, C.E., Burgess, E. et al. Pilot study of CyA in patients with symptomatic PBC. Gastroenterology 1988, 95: 1356-1363.

15. Triger, D.R., Manifold, I.H., Cloke, P. et al. D Penicillamine in PBC: 2 year results of a single centre doubleblind controlled trial. Gut 1980, 21: 919-920 (abstract).

16. Epstein, O., Jain, S., Lee, R.G. et al. D Penicillamine treatment improves survival in PBC. Lancet 1981, i: $1275-1277$. values over $100 \mu \mathrm{mol} / 1$ and/or other complications of chronic liver disease, particularly portal hypertension and deterioration in quality of life, and before the onset of hyponatraemia and significant bone disease she or he should be referred for assessment at a liver transplantation centre.
17. Matloff, D.S., Alpert, E., Resnick, R.H. \& Kaplan, M.M. A prospective trial of D penicillamine in PBC. $N$ Engl J Med 1982, 306: 319-26.

18. Bassendine, M.F., Macklon, A.F., Mulcahy, R. et al. Controlled trial of high and low dose $\mathrm{D}$ penicillamine in PBC. Gut 1982, 23: 909 (abstract).

19. Taal, B.G., Schalm, S.W., Tenkate, F.W. et al. Low therapeutic value of $D$ penicillamine in a short term prospective trial in primary biliary cirrhosis. Liver 1983, 3: $345-352$.

20. Bodenheimer, H.C., Schaffner, F., Sternlieb, I. et al. A prospective clinical trial of $\mathrm{D}$ penicillamine in the treatment of PBC. Hepatology 1985, 5: 1139-1142.

21. Dickson, E.R., Fleming, T.R., Wiesner, R.H. et al. Trial of penicillamine in advanced primary biliary cirrhosis. $N$ Engl J Med 1985, 312: 1011-1015.

22. Neuberger, J., Christensen, E., Portmann, B. et al. Double blind controlled trial of $\mathrm{D}$ pencillamine in patient with primary biliary cirrhosis. Gut 1985, 26: 114-119.

23. Mitchison, H.C., Watson, A.J., Bassendine, M.F.et al. A pilot double blind controlled trial of prednisolone treatment in primary biliary cirrhosis (PBC). J Hepatol 1986, 3: S28 (abstract).

24. Hadziyannis, S. \& Hadziyannis, E. A randomized controlled trial of UDCA in primary biliary cirrhosis (PBC). Hepatology 1988, 8: 1421 (abstract).

25. Beswick, D.R., Klatskin, G. \& Boyer, J.L. Asymptomatic primary biliary cirrhosis. A progress report on long term follow-up and natural history. Gastroenterology 1985, 89: 267-271.

26. Neuberger, J. Natural history of primary biliary cirrhosis. In : Tygstrup, N. \& Orlandi, F. (eds) Cirrhosis of the Liver: Methods and Fields of Research. Elsevier Science Publishers, Amsterdam, 1987, pp. 343-352.

27. Mitchison, H., Kelly, P., Neuberger, J., Bassendine, M., Williams, R. \& James, O. Symptoms development and prognosis in asymptomatic PBC. Hepatology 1988, 5: 1417 (abstract).

28. Balasubramaniam, K., Grambsch, P.N., Wiesner, R.H. et al. Asymptomatic primary biliary cirrhosis: patients have a diminished survival. Hepatology 1987, 7: 1025 (abstract).

29. Shapiro, J.M., Smith, H. \& Schaffner, S.F. Serum bilirubin: a prognostic factor in PBC. Gut 1979, 20: $137-140$.

30. Roll, J.R., Boyer, J.D., Barry, D. \& Klatskin, G. The prognostic importance of clinical and histological features in asymptomatic and presymptomatic primary biliary cirrhosis. $N$ Engl J Med 1983, 318: 1-7. 
31. Portmann, B., Popper, H., Neuberger, J. \& Williams, R. Sequential and diagnostic features in primary biliary cirrhosis based on serial histologic study in 209 patients. Gastroenterology 1985, 88: 1777-1190.

32. Rubel, L.R., Sabin, L., Seeff, L.B. et al. Does primary biliary cirrhosis in men differ from primary biliary cirrhosis in women. Hepatology 1984, 4: 671-677.

33. Lucey, M.R., Neuberger, J.M. \& Williams, R. Primary biliary cirrhosis in men. Gut 1986, 27: 1373-1376.

34. Malia, W.M., Johnson, P.J., Neuberger, J. et al. Hepatocellular carcinoma in primary biliary cirrhosis. Gastroenterology 1984, 84: 660-667.

35. Iwatsuki, S., Gordon, R.D., Shaw, B.W. \& Starzl, T.E. Role of liver transplantation in cancer therapy. Ann Surg 1985, 202: 401-407.

36. O’Grady, J.G., Polson, R.J., Rolles, K., Calne, R.Y. \& Williams, R. Liver transplantation for malignant disease. Ann Surg 1988, 207: 373-379.

37. Wolde, A.M., Schaffner, F., Kapelman, B. \& Sacks, H. Malignancy in primary biliary cirrhosis. Am J Med 1984, 76: $1075-1078$.

38. Goudie, B.M., Burt, A.D., Boyle, P. et al. Breast cancer in women with primary biliary cirrhosis. $\mathrm{Br} \mathrm{Med} J \mathrm{1985}$, 291: $1597-1598$.

39. Wiesner, R.H., Grambsch, P.M., Lindor, K.D., Ludwig, J. \& Dickson, E.R. Clinical and statistical analyses of new and evolving therapies for primary biliary cirrhosis. Hepatology 1988, 8: 668-676.

40. Epstein, O., Cook, D.G., Jain, S. et al. D Penicillamine in primary biliary cirrhosis - An untested and untestable treatment. Hepatology 1985, 1 (suppl): 49 (abstract).

41. Maddrey, W.C. \& Van Thiel, D.H. Liver transplantation: an overview. Hepatology 1988, 8: 948-959.

42. Esquivel, C.O., Van Thiel, D.H., Demetris, A.J. et al. Transplantation for primary biliary cirrhosis. Gastroenterology 1988, 94: 1207-1216.

43. Wiesner, R.H., Dickson, E.R., Hodgson, S.F. \& Wahner, $H$. The effect of liver transplantation on bone mineral density in PBC. Hepatology 1987, 7: 1049 (abstract).

44. Bismuth, H., Ericzon, B.G., Rolles, K. et al. Hepatic transplantation in Europe. First report of the European Liver Transplant Registry. Lancet 1987, ii: 674-677.

45. Dickinson, E.R., Grambsch, P., Markus, B.M., Klintmalm, B.H., Wiesner, R.H. \& Starzl, T.E. Transplantation markedly improves survival in PBC patients application of the Mayo model on Pittsburgh transplant patients. Gastroenterology 1988, 94: A535 (abstract).

46. Neuberger, J., Portmann, B., MacDougall, B.R. et al. Recurrence of primary biliary cirrhosis after liver transplantation. $N$ Engl J Med 1982, 306: 1-4.

47. Portmann, B., O'Grady, J. \& Williams, R. Disease recurrence following orthotopic liver transplantation. Transplant Proc 1986, 18: 136-141.

48. Van Thiel, D.H. \& Gavaler, J.S. Recurrent disease in patients with liver transplantation: when does it occur and how can we be sure? Hepatology 1987, 7: 181-183.

49. Haagsma, E.B., Klein, R., Huizenga, J. et al. Subtypes of antimitochondrial antibodies in primary biliary cirrhosis before and after orthotopic liver transplantation. Hepatology 1987, 7: 129-133.

50. McNeil, B.J., Weichselbaum, R. \& Pauker, S.G. Fallacy of the five year survival in lung cancer. $N$ Engl $J$ Med 1978, 299: 1397-1401.
51. Pauker, S.G. \& Kassierer, J.P. Decision analysis. A review. $N$ Engl J Med 1987, 316: 250-257.

52. Kaissirer, J.P., Moskowitz, A.J., Lau, L. \& Pauker, S.G. Decision analysis: a progress report. Ann Int Med 1987, 106: 275-291.

53. Christensen, E., Schlichting, P., Andersen, P.K. et al. Updating prognosis and therapeutic effect evaluation in cirrhosis using Cox's multiple regression model for time dependent variables. Scand J Gastroenterol 1986, 21: $163-174$.

54. Eriksson, S. \& Zettervall, O. The $\mathrm{N}$ terminal propeptide of collagen type III in serum as a prognostic indicator in primary biliary cirrhosis. J Hepatol 1986, 2: 370-378.

55. Babbs, C., Hunt, L.P., Naboubi, N.Y., Smith, A., Rowan, B.P. \& Warnes, T.W. Type III procollagen peptide: a marker of disease activity and prognosis in primary biliary cirrhosis. Lancet 1988, i: 1021-1024.

56. Nyberg, A., Engstrom-Laurent, A. \& Loof, L. Serum hyaluronate in primary biliary cirrhosis. A biochemical marker of progressive liver damage. Hepatology 1988, 8: $142-147$.

57. Smith, N.D. \& Boyer, J.L. Are mitochondrial antibodies of prognostic value in primary biliary cirrhosis? Hepatology 1986, 6: 739-741.

58. Cotting, J., Widmer, T., Bircher, et al. Accurate prediction of death by serial determination of galactose elimination capacity (GEC) in primary biliary cirrhosis. Hepatology 1987, 7: 1119 (abstract).

59. Neuberger, J., Altman, D., Polson, R. et al. Prediction of survival after transplantation for PBC an analysis of two centres. J Hepatol 1988, 7: S65 (abstract).

60. Cuervas-Mons, V., Millan, I., Gavaler, J.S., Starzl, T.E. \& van Thiel, D.H. Prognostic value of pre-operatively obtained clinical and laboratory data in predicting survival following orthotopic liver transplantation. Hepatology 1986, 6: 922-927.

61. Rimola, A., Gavaler, J.S., Schade, R.R., El-Lankany, S., Starzl, T.E. \& Van Thiel, D.H. Effects of renal impairment on liver transplantation. Gastroenterology 1987, 93: $148-156$.

62. Barasso Garcia de Silva, E., Gore, S.M., White, D.R.G. et al. Analysis of risk factors in liver transplantation. Transplant Proc 1986, 18: 1210-1212.

63. Wood, R.P., Shaw, B.W., Starzl, T.E. et al. Extrahepatic complications of liver transplantation. Semin Liver Dis 1985, 5: 377-384.

64. Adams, D.H., Ponsford, S., Gunson, B. et al. Neurological complications following liver transplantation. Lancet 1987, i: 949-951.

65. Adler, M., Gavaler, J.S., Duquesnoy, R. et al. Relationship between diagnosis, pre-operative evaluation and prognosis after orthotopic liver transplantation. Ann Surg 1988, 208: 196-202.

66. Degos, F., Samuel, D., Castaing, D., Benhamou, J.P. \& Bismuth, $H$. Selection criteria of patients with $\mathrm{PBC}$ for liver transplant. J Hepatol 1988, 7: S23 (abstract).

67. Beck, R., Pauker, S.G., Gottlieb, J.E., Klein, K. \& Kassirer, J.P. Convenient approximation of life expectancy ("Deale"). Use in medical decision making. Am J Med 1982, 73: 889-897. 